\title{
Composição química, atividade antimicrobiana e antioxidante do óleo essencial de
}

\author{
folhas Myrcia palustris DC. (MYRTACEAE)
}

Chemical composition, antimicrobial and antioxidant activity of leaves essential oil the Myrcia palustris DC. (MYRTACEAE)

Composición química, actividad antimicrobiana y antioxidante del aceite esencial de hojas de Myrcia palustris DC. (MYRTACEAE)

Camila Vogt dos Santos

ORCID: https://orcid.org/0000-0001-7566-8003 Universidade Estadual do Oeste do Paraná, Brasil

E-mail: cami_vogt@ hotmail.com

Ana Paula Mallman

ORCID: https://orcid.org/0000-0001-7568-8637 Universidade Estadual do Oeste do Paraná, Brasil E-mail: ap.mallmann@ hotmail.com

Adrieli Gorlin Toledo

ORCID: https://orcid.org/0000-0002-0199-8330 Universidade Estadual do Oeste do Paraná, Brasil E-mail: adrieligorlin@live.com

Débora Marina Bandeira

ORCID: https://orcid.org/0000-0001-5956-7210 Universidade Estadual do Oeste do Paraná- Brasil E-mail: dm-bandeira@hotmail.com

Willian Ferreira da Costa

ORCID: https://orcid.org/0000-0003-1593-5784 Universidade Estadual de Maringá, Brasil E-mail: wfcosta@uem.br

Daíse Miranda Ávila Marins

ORCID: https://orcid.org/0000-0002-1806-8375

Universidade Estadual de Maringá, Brasil E-mail:mirandadaise23@gmail.com

Juliana Moço Corrêa ORCID: https://orcid.org/0000-0003-4526-0060 Universidade Estadual do Oeste do Paraná, Brasil E-mail: juliana.correa2@unioeste.br

Fabiana Gisele da Silva Pinto ORCID: https://orcid.org/0000-0002-0486-8486 Universidade Estadual do Oeste do Paraná, Brasil E-mail: fabiana.pinto@unioeste

\begin{abstract}
Resumo
O Brasil apresenta a maior biodiversidade no mundo e neste contexto, suas espécies nativas tornam-se uma alternativa na busca por moléculas bioativas para bioprospecção de antimicrobianos e antioxidantes naturais. Pertencente à família Myrtaceae, a espécie Myrcia palustris DC, conhecida popularmente como pintagueira-do-mato, não apresenta estudos referente às suas atividades biológicas e composição química. Diante disso, o objetivo deste trabalho foi determinar a composição química do óleo essencial de $M$. palustris por cromatografia gasosa acoplada à espectrometria de massas (CG-EM), avaliar sua atividade antimicrobiana pela técnica de microdiluição em caldo e a atividade antioxidante pela técnica de 2,2-difenil-1-picril-hidrazila (DPPH). O rendimento total a partir da extração pela técnica de hidrodestilação do óleo essencial foi de 0,35\%. A CG-EM revelou a presença de 28 compostos, sendo a maioria da classe dos sesquiterpenos. A atividade antimicrobiana foi observada para todas as bactérias Grampositivas (Bacillus subtilis, Enterococcus faecalis, Staphylococcus aureus) com exceção de Staphyloccocus epidermidis. Em relação às Gram-negativas, verificou-se atividade inibitória apenas para Shigella flexneri. Para levedura Candida albicans não foi observada atividade inibitória e nem fungicida. O óleo essencial apresentou capacidade de redução de radicais de DPPH até 82,81\%, confirmando seu potencial antioxidante. Sugere-se que a ação antimicrobiana e antioxidante presentes no óleo essencial de $M$. palustris esteja relacionada à presença dos compostos majoritários $\alpha$-Guaieno (25,89\%), $\alpha$-Bulneseno $(13,39 \%)$ e $\beta$-Selineno $(4,76 \%)$.
\end{abstract}


Palavra-chave: Atividades biológicas; Pitangueira-do-mato; substâncias bioativas; DPPH.

\begin{abstract}
Brazil has the greatest biodiversity in the world and in this context, its native species become an alternative in the search for bioactive molecules for bioprospecting antimicrobials and natural antioxidants. Belonging to the Myrtaceae family, the species Myrcia palustris DC, popularly known as pintagueira-do-mato, does not present studies regarding its biological activities and chemical composition. Therefore, the objective of this work was to determine the chemical composition of the essential oil of M. palustris by gas chromatography coupled with mass spectrometry (GC-MS), to evaluate its antimicrobial activity by the broth microdilution technique and the antioxidant activity by the broth technique. 2,2-diphenyl-1-picryl-hydrazyl (DPPH). The total yield from the essential oil hydrodistillation technique was $0.35 \%$. The GC-MS revealed the presence of 28 compounds, the majority being from the sesquiterpenes class. Antimicrobial activity was observed for all Gram-positive bacteria (Bacillus subtilis, Enterococcus faecalis, Staphylococcus aureus) with the exception of Staphyloccocus epidermidis. Regarding Gram-negatives, there was an inhibitory activity only for Shigella flexneri. For yeast Candida albicans, no inhibitory or fungicidal activity was observed. The essential oil showed a capacity to reduce DPPH radicals up to $82.81 \%$, confirming its antioxidant potential. It is suggested that the antimicrobial and antioxidant action present in the essential oil of $M$. palustris is related to the presence of the major compounds $\alpha$-Guaieno (25.89\%), $\alpha$-Bulnesene $(13.39 \%)$ and $\beta$-Selinene $(4,76 \%)$.
\end{abstract}

Keywords: Biological activities; Pitangueira-do-mato; bioactive substances; DPPH.

\title{
Resumen
}

Brasil tiene la mayor biodiversidad del mundo y en este contexto, sus especies nativas se convierten en una alternativa en la búsqueda de moléculas bioactivas para bioprospección de antimicrobianos y antioxidantes naturales. Perteneciente a la familia Myrtaceae, la especie Myrcia palustris DC, conocida popularmente como pintagueira-domato, no presenta estudios sobre sus actividades biológicas y composición química. Por tanto, el objetivo de este trabajo fue determinar la composición química del aceite esencial de $M$. palustris mediante cromatografía de gases acoplada a espectrometría de masas (GC-MS), para evaluar su actividad antimicrobiana mediante la técnica de microdilución en caldo y la actividad antioxidante mediante la técnica 2,2-difenil-1-picril-hidrazilo (DPPH). El rendimiento total de la técnica de hidrodestilación de aceites esenciales fue del 0,35\%. CG-EM reveló la presencia de 28 compuestos, la mayoría de los cuales pertenecen a la clase de sesquiterpenos. Se observó actividad antimicrobiana para todas las bacterias grampositivas (Bacillus subtilis, Enterococcus faecalis, Staphylococcus aureus) con la excepción de Staphyloccocus epidermidis. En cuanto a los gram negativos, hubo actividad inhibidora solo para Shigella flexneri. Para la levadura Candida albicans, no se observó actividad inhibidora o fungicida. El aceite esencial mostró capacidad para reducir los radicales DPPH hasta en un 82,81\%, lo que confirma su potencial antioxidante. Se sugiere que la acción antimicrobiana y antioxidante presente en el aceite esencial de $M$. palustris está relacionada con la presencia de los compuestos principales $\alpha$-Guaieno (25,89\%), $\alpha$-Bulneseno $(13,39 \%)$ y $\beta$-Selineno $(4,76)$. \%).

Palabra clave: Actividades biológicas; Pitangueira-do-mato; sustancias bioactivas; DPPH.

\section{Introdução}

Nos últimos anos é cada vez maior a procura por produtos que possam substituir os sintéticos (antimicrobianos e antioxidantes) nas indústrias alimentícia, farmacêutica e cosmética, isso porque o consumidor está se tornando cada vez mais criterioso e exigente quanto à qualidade dos produtos que consome. Além disso, os produtos sintéticos comumente utilizados vêm causando inúmeros problemas tanto para o meio ambiente quanto para animais e humanos, em decorrência do seu uso inadequado e das elevadas concentrações em que são aplicados. (Silva et al., 2018; Machado \& Junior, 2011)

Ademais, o uso exacerbado de antimicrobianos tem selecionado a presença de microrganismos resistentes, reforçando a preocupação das indústrias em usar produtos que sejam naturais, isso porque a maioria dos produtos provenientes de espécies vegetais são menos agressivos ao ambiente e com menor toxicidade para o homem e ambiente. (Ostrosky et al., 2008; Packer \& Luz, 2007; da Silva et al., 2007)

Por sua vez, a substituição de antioxidantes sintéticos também se faz necessário, uma vez que um dos maiores problemas enfrentados pelas indústrias é oxidação lipídica, que provoca sabores e odores indesejáveis nos produtos, e pode levar à formação de compostos potencialmente tóxicos (Santos et al., 2020) e para sanar estes problemas são utilizados antioxidantes sintéticos. Os mais comuns são butil-hidroxi-tolueno (BHT), butil-hidroxi-anisol (BHA), terc-butil-hidroxquinona (TBHQ), tri-hidroxi-butil-fenona (THBP) e galato de propila (GP) e essas substâncias já despertam preocupação 
referente às doses utilizadas, podendo apresentar também efeitos tóxicos. Além disso, há evidências de que radicais livres e outros antioxidantes são os grandes responsáveis por causar inúmeras doenças degenerativas e cardiovasculares, bem como declínio do sistema imune e disfunções cerebrais.

Desta maneira, estudos para descobertas de antioxidantes e antimicrobianos de origem natural já estão sendo realizadas, justificando a importância de estudos para descoberta de moléculas capazes de sequestrar radicais livres e inibir o crescimento de microrganismos resistentes e possam ser utilizadas em conjunto para resolver essa problemática. (Degáspari \& Waszczynskyj, 2004; Gonçalves et al., 2015; Melo et al., 2011; Souza et al., 2007)

O Brasil é o local mais rico em biodiversidade no mundo, tornando-se muito importante na busca de novos princípios ativos, fornecendo matérias-primas para obtenção de novos produtos químicos. Muitas espécies vegetais nativas já servem como fonte de pesquisas a fim de descobrir suas atividades biológicas. (Rosa et al., 2016)

Nesse sentido, os óleos essenciais (OEs) presentes em plantas aromáticas, são substâncias provenientes do metabolismo secundário, constituídas por substâncias voláteis, e seus compostos orgânicos são as principais substâncias com ação terapêutica (Almeida, 2015; Becker et al., 2017; Rosa et al., 2016). Recentemente, foram reconhecidos como poderosos antioxidantes naturais, podendo ser utilizados como substitutos em potenciais aos antioxidantes sintéticos na indústria alimentícia e farmacêutica, além disso, representam uma abordagem interessante contra a ocorrência da resistência de patógenos microbianos aos medicamentos atuais. (Bozin et al., 2007; Mimica-Dukic et al., 2004; Solórzano-Santos \& Miranda-Novales, 2012)

Dentre as muitas espécies que apresentam atividades biológicas, as da família Myrtaceae destacam-se por ser uma das mais abundantes do ecossistema brasileiro, e ainda por apresentarem um alto teor de óleo essencial, que são muito estudados e testados em várias atividades biológicas (Silva et al., 2018). O gênero Myrcia é bastante interessante do ponto de vista químico e farmacológico, e se destaca pelo seu potencial terapêutico, além disso, muitas espécies já apresentam potencial citotóxico, antioxidante, antiflamatória e analgésica (Stefanello et al., 2011). Dentre todas as atividades já descritas para o gênero Myrcia, as mais relatadas são em relação à diabetes comprovando seu uso na medicina popular. Conhecidas como "planta insulina", as espécies M. multiflora, M. salcifolia e M. shaerocarpa são muito usadas no tratamento desta doença. (Russo et al., 1990)

Conhecida popularmente como pitangueira-do-mato, murta-do-brejo e baga-de-sabiá, a espécie nativa M. palustris DC. é encontrada principalmente no sul do Brasil e destaca-se na pesquisa por novos compostos que possam substituir os antimicrobianos e o antioxidantes sintéticos que são atualmente utilizados.

Tendo em vista os problemas relacionados à resistência microbiana e aos processos oxidativos, o objetivo deste estudo foi determinar a composição química do $\mathrm{OE}$ das folhas de $M$. palustris, bem como avaliar sua atividade antimicrobiana e determinar o potencial antioxidante.

\section{Metodologia}

A pesquisa foi realizada em condições controladas in vitro e aplicou-se o método quantitativo. Como existem dúvidas sobre a ação do óleo essecial sobre bactérias patogênicas, optou-se pelo método hipotético-dedutivo (Pereira et al., 2018).

\subsection{Coleta, secagem e identificação da planta:}

Os experimentos foram realizados no laboratório de Microbiologia e Biotecnologia (LAMIBI), situado na Universidade Estadual do Oeste do Paraná - UNIOESTE. As folhas de M. palustris foram coletadas no Parque Ecológico

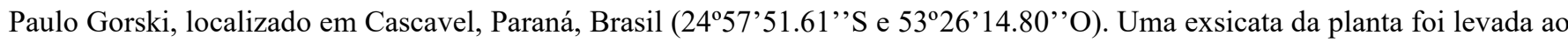
Herbário UNOP para identificação botânica e registro do voucher, sob o número UNOP 8915. Após as coletas, as folhas foram secas a $40{ }^{\circ} \mathrm{C}$ e moídas em moinho de facas tipo willye, com membrana $0,42 \mathrm{~mm}$ de granulometria e, posteriormente, o pó 
obtido foi armazenado em frasco de vidro fechado ao abrigo da luz, em temperatura ambiente até a extração do óleo essencial. (Weber et al., 2014)

\subsection{Extração do Óleo essencial:}

Para a extração do óleo essencial $140 \mathrm{~g}$ do material vegetal foi adicionado a 1,4 L de água destilada, de acordo com Weber et al., (2014) a solução foi colocada em aparelho Clevenger seguindo a técnica de hidrodestilação por aproximadamente 5 horas. O rendimento do OE foi expresso em porcentagem na relação massa/volume pela medida de densidade. Para realização dessa medida, foi utilizado um picnômetro de $1,0 \mathrm{~mL}$, previamente seco, tarado e aferido. Após essa etapa, observou-se o volume $(\mathrm{mL})$ de $\mathrm{OE}$ obtido após a extração do óleo por massa $(\mathrm{g})$ de material vegetal descrita pela quarta edição da Farmacopeia Brasileira (BRASIL, 2019). O óleo extraído foi armazenado em freezer a $4{ }^{\circ} \mathrm{C}$ até o momento da utilização.

\subsection{Cromatografia de gasosa acoplada à Espectrometria de Massas GC-MS:}

As análises dos compostos do óleo essencial de M. palustris foram realizadas a partir do sistema Thermo-Finnigan CG-EM. A separação dos compostos foi realizada utilizando GC FOCUS (Thermo Electron), acoplado a um espectrômetro de massas DSQ II (Thermo Electron) e um injetor automático TriPlus (Thermo Electron), utilizando uma coluna capilar de sílica fundida HP-5ms (30m comprimento, 0,25 e 0,25 um ID do filme; composição 5\% fenil-95\% de dimetilpolissiloxano). A temperatura do injetor foi de $250^{\circ} \mathrm{C}$. A amostra e os padrões de alcano C7-C28 foram injetados em uma razão de divisão 1:25. A programação da temperatura utilizada foi de $50^{\circ} \mathrm{C}$ mantida por $2 \mathrm{~min}$, um aumento de temperatura para $180^{\circ} \mathrm{C}$ na proporção de $2^{\circ} \mathrm{C} \mathrm{min}{ }^{-1}$, seguido por um aumento para $290^{\circ} \mathrm{C}$ na proporção de $5^{\circ} \mathrm{C} \mathrm{min.}{ }^{-1} \mathrm{~A}$ interface entre o $\mathrm{GC}$ e a MS foi mantida a $270^{\circ} \mathrm{C}$ e a temperatura da fonte de ionização do espectrometro de massa foi de $250^{\circ} \mathrm{C}$. A identificação dos compostos foi realizada comparando seus tempos de retenção com os tempos de retenção obtidos da literatura e seus índices de retenção. (Adams, 2007; Amorim et al., 2019; Babushok et al., 2011; Yu et al., 2007)

\subsection{Microrganismos utilizados e preparo do inóculo}

Os microrganismos utilizados são das coleções Americam Type Culture Collection (ATCC) e Cefar Diagnóstica (CCD), sendo as bactérias Gram positivas: Bacillus subtilis (CCD-04), Enterococcus faecalis (ATCC 19433), Staphylococcus aureus (ATCC 25923), S. epidermidis (ATCC 12228); Gram negativas: Aeromonas hydrophila (ATCC 7966), Escherichia coli (ATCC 25922), Klebsiella pneumoniae (ATCC 13883), Pseudomonas aeruginosa (ATCC 27853), Proteus mirabilis (ATCC 25933), Salmonella Enteritidis (ATCC 13076), Salmonella Galinarum (ATCC 1138), Salmonella Heidelberg (ATCC), Salmonella Typhimurium (ATCC 14028), Shigella flexeri (ATCC 12022) e também a levedura Candida albicans (ATCC 12031). Os microrganismos foram recuperados em Caldo Infusão de Cérebro e Coração (BHI), durante 24 horas a $36^{\circ} \mathrm{C}$, em seguida, semeado em placa de Petri contendo ágar Muller-Hinton $(\mathrm{MH})$ e incubados durante 24 horas a $36{ }^{\circ} \mathrm{C}$. Para realização dos experimentos, a concentração dos microrganismos foi ajustada em solução salina $0,85 \%$ para $1 \times 10^{5} \mathrm{UFC} / \mathrm{mL}$ para bactérias e $1 \times 10^{6} \mathrm{UFC} / \mathrm{mL}$ para levedura C. albicans.

\subsection{Atividade Antimicrobiana - Determinação da Concentração Inibitória Mínima (CIM)}

A atividade antimicrobiana do óleo essencial de M. palustris foi avaliada seguindo as normas do Clinical and Laboratory Standards Institute (CLSI, 2015) e Scur et al., (2014) com modificações e a concentração Inibitória Mínima (CIM) foi realizada pelo método de microdiluição em caldo. O óleo essencial foi solubilizado em metanol (P.A), filtrado com membrana filtrante e diluído em caldo MH para bactérias e em Roswell Park Memorial Institute (RPMI-1640) para a levedura C. albicans. Na placa de microdiluição de 96 poços foram dispensados $150 \mu \mathrm{L}$ de MHB ou RPMI-1640 caldo, e em seguida, 
diluições seriadas do óleo essencial foram realizadas nas concentrações de 7000-3,41 $\mu \mathrm{g} / \mathrm{mL}$. Por fim, $10 \mu \mathrm{L}$ do inóculo foram adicionados a cada poço e incubados a $36^{\circ} \mathrm{C}$ por 24 horas. Para o controle positivo foram utilizados antibiótico comercial Gentamicina (200 mg/mL) e o antifungico comercial Nistadina $(200 \mathrm{mg} / \mathrm{mL})$. Para comprovar a viabilidade do microrganismo, um controle negativo foi feito, dispensando no poço apenas $150 \mu \mathrm{L}$ MHB ou RPMI-1640 e $10 \mu \mathrm{L}$ do inóculo. Para interpratação dos resultados, foi utilizado $25 \mu \mathrm{L}$ de Cloreto de Trifeniltetrazólio (TTC) a $0,5 \%$, atuando como um revelador colorimétrico, os poços que apresentaram colocaração vermelha obtiveram resposta negativa para inibição do crescimento bacteriano. A CIM foi realizada em triplicada sendo possível determinar a menor concentração do óleo essencial capaz de inibir o crescimento microbiano.

\subsection{Determinação da Concentração Bactericida Mínima (CBM)/Concentração Fungica Mínima (CFM)}

Antes da adição do TTC 0,5\% para determinação da CIM foi retirada uma alíquota de $2 \mu \mathrm{L}$ de cada poço e transferida individualmente para placas de Petri contendo $\mathrm{MH}$ agar, que foram incubadas por 24 horas a $36^{\circ} \mathrm{C}$. Para determinar $\mathrm{CBM}$ ou CFM, ou seja, a menor concentração do oleo essencial capaz de causar a morte do microrganismo, foi observado o crescimento de colônia microbiana na placa (Scur et al., 2014). A CIM e CBM do óleo essencial foram classificados de acordo com os critérios propostos por Sartoratto et al., (2004). A atividade foi classificada em alta $(<100 \mu \mathrm{g} / \mathrm{mL}$ ), moderada (entre 100 e 500 $\mu \mathrm{g} / \mathrm{mL}$ ), baixa (entre 500 e $1000 \mu \mathrm{g} / \mathrm{mL}$ ) e muito baixa (acima de $1000 \mu \mathrm{g} / \mathrm{mL}$ ). O ensaio foi realizado em triplicata.

\subsection{Atividade Antioxidante}

O teste da atividade antioxidante do oleo essencial foi realizado pelo método de redução do DPPH, proposto por Rufino et al., (2007) e Weber et al., (2014). Foi realizada uma curva de calibração (0, 10, 20, 30, 40, 50 e $60 \mu \mathrm{M}$ de DPPH) para obter a concentração do DPPH no meio após a reação com o óleo essencial, com a equação $y=0,0113 x-0,0429(R 2=0,995)$, onde $y$ é a concentração de DPPH e $x$ a absorbância. Em seguida, alíquota de $0,1 \mathrm{~mL}$ do oleo essencial em diferentes concentrações $(35,75,150,300,600 \mathrm{mg} / \mathrm{mL})$ foram adicionados a 3,9 $\mathrm{mL}$ da solução de metanólica de DPPH $(60 \mathrm{mM})$ e homogeneizadas em tubo agitador. Como controle negativo $0,1 \mathrm{~mL}$ de Metanol foi adicionado a 3,9 mL de DPPH, e como controle positivo, o antioxidante sintético BHT (butil-hidroxi-tolueno) foi utilizado em diferentes concentrações $(1,0,50,0,25,0,10$ e $0,05 \mathrm{mg} / \mathrm{mL})$. Os testes foram realizados no escuro, utilizando espectofotômetro a $515 \mathrm{~nm}$ para as leituras de 1 min até a estabilização da absorbância. O metanol P.A. foi utlizado para calibração do aparelho. A porcentagem de sequestro de radicais livres (AA\%) foi expressa pela equação: $A A \%=\frac{\left(A_{0}-A_{1}\right)}{A_{1}}$ x100, onde $A_{0}$ é a absorbância do controle negativo e $A_{l}$ é a absorbância da amostra. Para o cálculo do $\mathrm{IC}_{50}$ (quantidade de substância antioxidante necessária para reduzir em 50\% a concentração inical de DPPH), as concentrações do óleo essencial e BHT foram utilizadas para obter a equação da linha com $\mathrm{R}^{2}$ maior que 0,80 , e assim encontrar o valor de $\mathrm{IC}_{50}$, a partir da regreção linear. Os testes foram realizados em triplicada e expressos como media \pm desvio padrão.

\section{Resultados e Discussão}

\subsection{Composição química do óleo essencial de M. palustris}

Verificou-se que as folhas de M. palustris apresentaram rendimento de 0,35\%. Dentre as espécies do gênero, $M$. palustris apresentou um baixo rendimento quando comparada com M. sylvativa (0,5\%) (Rosa et al., 2016) e M. tumosa com (0,54\%) (Sá et al., 2012), mesmo assim este valor é considerado aceitável para um potencial cultivo industrial. (Henriques et al., 1997) 
A composição química do óleo essencial das folhas de M. palustris revelou a presença de 29 compostos (Tabela 1), representando $80,75 \%$ da área total da amostra analisada. Os compostos majoritários encontrados no OE foram os sesquiterpenos $\alpha$-Guaieno (25,89\%), $\alpha$-Bulneseno (13,39\%), seguido de $\beta$-Selineno (4,76\%).

Tabela 1 - Composição química do óleo essencial das folhas de M. palustris obtido por hidrodestilação e analisado por $C G$ $E M$.

\begin{tabular}{|c|c|c|c|c|c|}
\hline $\mathrm{N}^{\mathrm{o}}$ & Composto & $\mathrm{TR}$ & Área (\%) & IR & $\mathrm{IR}^{*}$ \\
\hline 1 & $\alpha$-Cubebeno & 25,35 & 0,18 & 1344 & 1345 \\
\hline 2 & $\alpha$-Copaeno & 26,57 & 0,77 & 1372 & 1376 \\
\hline 3 & $\beta$-Bourboneno & 26,90 & 0,27 & 1379 & 1381 \\
\hline 4 & $\beta$-Elemeno & 27,19 & 2,71 & 1386 & 1390 \\
\hline 5 & $\alpha$-Gurjuneno & 27,91 & 0,23 & 1403 & 1409 \\
\hline 6 & Cariofileno & 28,41 & 3,38 & 1415 & 1419 \\
\hline 7 & $\beta$-Gurjuneno & 28,84 & 0,44 & 1430 & 1431 \\
\hline 8 & $\alpha$-Guaiaeno** & 29,16 & 25,89 & 1433 & 1437 \\
\hline 9 & $\alpha$-Humuleno & 29,90 & 0,65 & 1450 & 1453 \\
\hline 10 & Aromadendreno & 30,08 & 0,30 & 1455 & 1460 \\
\hline 11 & $\gamma$-Muuroleno & 30,75 & 1,30 & 1471 & 1476 \\
\hline 12 & Germacreno D & 30,98 & 3,13 & 1476 & 1480 \\
\hline 13 & $\beta$-Selineno** & 31,29 & 4,76 & 1484 & 1486 \\
\hline 14 & Viridifloreno & 31,40 & 0,97 & 1486 & 1492 \\
\hline 15 & $\alpha$-Selineno & 31,59 & 4,50 & 1491 & 1493 \\
\hline 16 & $\alpha$-Muuroleno & 31,74 & 1,09 & 1494 & 1498 \\
\hline 17 & $\alpha$-Bulneseno** & 31,87 & 13,39 & 1498 & 1504 \\
\hline 18 & $\gamma$-Cadineno & 32,30 & 1,02 & 1508 & 1513 \\
\hline 19 & $\delta$-Cadineno & 32,53 & 3,04 & 1514 & 1514 \\
\hline 20 & cis-calameno & 32,63 & 0,55 & 1517 & 1523 \\
\hline 21 & $\alpha$-Calacoreno & 33,40 & 0,47 & 1536 & 1540 \\
\hline 22 & Espatulenol & 34,78 & 4,52 & 1571 & 1576 \\
\hline 23 & Óxido de Cariofileno & 34,96 & 1,12 & 1575 & 1581 \\
\hline 24 & Globulol & 35,13 & 1,25 & 1579 & 1582 \\
\hline 25 & epi-Globulol & 35,45 & 0,59 & 1587 & 1585 \\
\hline 26 & iso-espatulenol & 36,94 & 0,83 & 1626 & 1633 \\
\hline 27 & epi- $\alpha$-cadinol & 37,32 & 0,52 & 1636 & 1638 \\
\hline 28 & epi- $\alpha$-muurolol & 37,39 & 0,91 & 1638 & 1641 \\
\hline 29 & $\alpha$-Cadinol & 37,81 & 1,97 & 1649 & 1652 \\
\hline 30 & $\mathrm{NI}$ & & 10,10 & & \\
\hline
\end{tabular}




\begin{tabular}{lcc}
\hline Total de Compostos & 90,85 & $80,75 \%$ \\
\hline **Compostos majoritários; NI: Compostos Não Identificados; TR: Tempo de Retenção; IR: Índice de Retenção; IR*: Índice \\
de Retenção encontrado na literatura. Fonte: Autores.
\end{tabular}
de Retenção encontrado na literatura. Fonte: Autores.

Dentro da família Myrtaceae, o gênero Myrcia é o mais estudado, apresentando cerca 16 espécies analisadas, e assim como em M. palustris, a presença de sesquiterpenos foi predominante em 15 delas, sendo que em M. acuminatissima e M. bombycina o conteúdo de monoterpenos foi ligeiramente maior que o de sesquiterpenos (Cerqueira et al., 2009). O OE de $M$. oblongata apresenta como principal composto o óxido de cariofileno (sesquiterpeno) (Santana et al., 2018), também identificado em nosso estudo no OE de M. palustris, mas em menor quantidade. O terceiro principal composto também identificado foi sesquiterpeno, $\beta$-Selineno, já foi observado em outras espécies do gênero como M. olingatha, M. lajeana e M. hatschbachii (Limberger et al., 2004). O composto majoritário encontrado aqui, $\alpha$-Guaieno, não foi identificado em nenhuma outra espécie do gênero Myrcia.

Observou-se que o perfil químico do OE de M. palustris diferiu em quantidade, número de compostos e conformação molecular entre as espécies do mesmo gênero. Essa variação pode ser explicada a condições ambientais distintas, tais como: solo, temperatura, irrigação, forma e época do plantio, intensidade das radiações solares, interações com polinizadores e predadores. (Nascimento et al., 2007; Scherer et al., 2009) Ademais, técnicas de extração e proveniência do material da planta (fresco ou seco) também podem interferir nos compostos químicos dos óleos essenciais, influenciando diretamente em suas propriedades funcionais, como atividades antioxidante e antimicrobiana (Martins Dias de Cerqueira et al., 2009; Gobbo-Neto \& Lopes, 2007)

\subsection{Atividade antimicrobiana - CIM/CBM(CFM)}

Os resultados de CIM e CBM do óleo essencial de M. palustris variaram de 1750 a 218,75 $\mu \mathrm{g} / \mathrm{mL}$ (tabela 2). Com exceção de $S$. epidermidis foi observada atividade para as outras cepas Gram-positivas, o melhor resultado apresentado foi para B. subtilis e $S$. aureus com CIM e CBM de 437,5 e $875 \mu \mathrm{g} / \mathrm{mL}$, respectivamente, considerado uma atividade moderada. Para $E$. faecalis o OE apresentou atividade antimicrobiana de baixa a moderada com CIM de 437,5 e CBM de $1750 \mu \mathrm{g} / \mathrm{mL}$. Dentre as bactérias Gram negativas, foi observada atividade inibitória moderada do OE contra $S$. flexeri, com CIM de $218,75 \mu \mathrm{g} / \mathrm{mL}$. Para a levedura C. albicans o OE não apresentou atividade antimicrobiana (Pandini et al., 2015; Sartoratto et al., 2004).

Apesar de não ter sido encontrado nenhum relato da atividade antimicrobiana do óleo essencial de M. palustris na literatura, foi observado dentro do gênero Myrcia espécies com propriedades antimicrobianas, como M. myrtifolia (Cerqueira et al., 2007), M. fosteri e M. ovata (Tenório et al., 2011), M. tomentosa (Sá et al., 2012) e M. oblongata (Santana et al., 2018).

A atividade antimicrobiana do OE de M. palustris pode ser devido a presença de compostos majoritários $\alpha$-Guaieno, $\alpha$-Bulneseno e $\beta$-Selineno. Esses compostos são sesquiterpenos e já é bem estabelecido o fato de que muitos terpenóides quando presentes em OEs apresentem efeitos antimicrobianos. Além disso, deve-se levar em consideração que OE é uma mistura complexa e também pode ocorrer interação sinérgica de todos os compostos, mesmo aqueles encontrados em concentrações muito baixas, mas a ação conjunta contribui com sua eficiência. (Claeson et al., 1992; Jiménez et al., 2012; Lin et al., 2012)

Observou-se que as bactérias Gram positivas foram mais suscetíveis ao OE de M. palustris do que as Gram negativas. Resultado semelhante foi verificado para o OE das espécies $M$. tomentosa e $M$. oblongata que apresentaram atividade antimicrobiana moderada para B. subtilis, S. aureus e E. faecalis (Sá et al., 2012; Santana et al., 2018). Diversos estudos já demonstraram que bactérias Gram-positivas são mais suscetíveis aos OEs quando comparadas as Gram-negativas, provavelmente devido à presença de compostos lipofílicos nos OEs, que promovem dano direto na membrana celular, 
causando seu rompimento. Além disso, afeta também a manutenção do pH e o bloqueio de enzimas bacterianas (Lang \& Buchbauer, 2012; Xavier et al., 2016).

Para C. albicans o OE não apresentou atividade, ao buscar resultados semelhantes na literatura encontramos o $\mathrm{OE}$ da espécie M. oblongata, que apresentou atividade considerada baixa, com CIM e CFM de $3500 \mu \mathrm{g} / \mathrm{mL}$ (Santana et al., 2018). A baixa eficiência do OE para leveduras pode ser justificada, pois os seus constituintes químicos do OE apresentar baixa hidrofobicidade, e por isso não conseguem interagir com os componentes lipídicos, sendo impedidos de penetrar na célula. (Castro \& Lima, 2010).

Tabela 2 - Concentração Inibitória Mínima (CIM) e Concentração Bactericida Mínima (CBM) do óleo essencial das folhas de M. palustris.

\begin{tabular}{|c|c|}
\hline Microrganismos & $\begin{array}{c}\text { Óleo Essencial } \\
\text { CIM/CBM }(\mu \mathrm{g} / \mathrm{mL})\end{array}$ \\
\hline \multicolumn{2}{|l|}{$\operatorname{Gram}(+)$} \\
\hline B. subtilis & $437,5 / 875$ \\
\hline E. faecalis & $437,5 / 1750$ \\
\hline S. aureus & $437,5 / 875$ \\
\hline S. epidermidis & NA/NA \\
\hline \multicolumn{2}{|l|}{ Gram (-) } \\
\hline A.hydrophila & $\mathrm{NA} / \mathrm{NA}$ \\
\hline E. coli & NA/NA \\
\hline K. pneumoniae & NA/NA \\
\hline P. aeruginosa & NA/NA \\
\hline P. mirabilis & NA/NA \\
\hline S. Enteritidis & $\mathrm{NA} / \mathrm{NA}$ \\
\hline S. flexeri & $218,75 / \mathrm{NA}$ \\
\hline S. Gallinarum & NA/NA \\
\hline S. Heidelberg & NA/NA \\
\hline S. Typhimurium & NA/NA \\
\hline \multicolumn{2}{|l|}{ Levedura } \\
\hline C. albicans & NA/NA \\
\hline
\end{tabular}

NA: sem atividade. Fonte: Autores.

\subsection{Atividade antioxidante}

A redução dos radicais livres pelo $\mathrm{OE}$ foi dependente das concentrações utilizadas, sendo o melhor resultado expresso na concentração de $100 \mathrm{mg} / \mathrm{mL}$, apresentando a maior porcentagem de sequestro de DPPH de 82,81\% e valor de $\mathrm{IC}_{50}$ 255,97 $\pm 3,2$ (Tabela 3 e 4). Esse valor de $\mathrm{IC}_{50}$ foi considerado estatisticamente diferente do valor encontrado para controle BHT, com $\mathrm{IC}_{50} 0,28 \pm 0,0 \mathrm{mg} / \mathrm{mL}$ (teste $\chi^{2}=252,8 ; \mathrm{GL}=1, \mathrm{p}<0.05$ ). Isso demonstra a necessidade de utilizar uma maior 
concentração de OE para reduzir a mesma quantidade de radicais livres DPPH quando comparado com o produto sintético comercial (BHT).

Tabela 3 - Valor de $\mathrm{IC}_{50}$ pelo ensaio do DPPH do óleo essencial das folhas de Myrcia palustris.

\begin{tabular}{llll}
\hline Amostra & $\mathbf{I C}_{\mathbf{5 0}}(\mathbf{m g} / \mathbf{m L})$ & Equação & $\mathbf{R}^{\mathbf{2}}$ \\
\hline BHT & $0,28 \pm 0,0$ & $\mathrm{y}=76,571 \mathrm{x}+28,47$ & 0,91 \\
OE de M. palustris & $255,97 \pm 3,2$ & $\mathrm{y}=0,104 \mathrm{x}+23,37$ & 0,96 \\
\hline
\end{tabular}

Média \pm desvio padrão. Fonte: Autores.

Tabela 4 - Porcentagem de sequestro de radicais DPPH do óleo essencial das folhas de M. palustris.

\begin{tabular}{lll}
\hline $\begin{array}{l}\text { Concentração } \\
(\mathbf{m g} / \mathbf{m L})\end{array}$ & $\begin{array}{l}\text { Controle } \\
\text { BHT }\end{array}$ & $\begin{array}{l}\text { OE de } \\
\text { M. palustris }\end{array}$ \\
\hline $\mathbf{1 0 0}$ & - & $82,81 \pm 1,0$ \\
$\mathbf{5 0}$ & - & $59,28 \pm 0,0$ \\
$\mathbf{2 5}$ & - & $42,75 \pm 0,1$ \\
$\mathbf{1 0}$ & - & $31,18 \pm 0,5$ \\
$\mathbf{5}$ & - & $21,49 \pm 0,0$ \\
$\mathbf{1}$ & $98,75 \pm 0,2$ & - \\
$\mathbf{0 , 5 0}$ & $77,23 \pm 0,1$ & - \\
$\mathbf{0 , 2 5}$ & $53,48 \pm 1,3$ & - \\
$\mathbf{0 , 1 0}$ & $38,03 \pm 0,2$ & - \\
$\mathbf{0 , 0 5}$ & $20,35 \pm 3,0$ & - \\
\hline
\end{tabular}

(-) Não testado; média \pm desvio padrão. Fonte: Autores.

Estudos sobre potencial antioxidante com espécies do gênero Myrcia demonstraram atividade antioxidante moderada, como é o caso de M. bella, M. tomentosa, M. lingua, M. splendens (Magina et al., 2010) e M. oblongata (Santana et al., 2018). Já com relação à espécie M. palustris não foram encontrados na literatura dados sobre atividade antioxidante de seu OE.

Em suma, plantas da família Myrtaceae apresenta grande capacidade em acumular substâncias fenólicas, já relatados na literatura como capazes de inibir os radicais livres presente no meio, sendo mais potentes que vitamina $\mathrm{C}$, vitamina $\mathrm{E}$ e carotenoides (Morais et al., 2009; Rice-Evans et al., 1996; Rosa et al., 2016; Takao et al., 2015). A atividade antioxidante atribuída aos compostos fenólicos está relacionada às suas propriedades redutoras e com a sua estrutura química, as quais desempenham importante papel no sequestro dos radicais livres, podendo agir na fase inicial e também na propagação do processo oxidativo (Souza et al., 2007). Porém, alguns OE apresentam comportamento antioxidante, conforme a estrutura química de seus compostos como, por exemplo, os terpenos que estão presentes em grandes quantidades no OE de $M$. palustris. (Amorati et al., 2013) 


\section{Conclusão}

A partir dos resultados obtidos neste estudo pode-se concluir que a composição química do OE de M. palustris revelou a presença de 29 compostos, os principais identificados são da classes dos sesquiterpetos: $\alpha$-Guaieno (25,89\%), $\alpha$ Bulneseno (13,39\%), seguido de $\beta$-Selineno (4,76\%), sendo o composto majoritário $\alpha$-Guaieno não encontrado em nenhuma outra espécie do gênero. Foi observada atividade antimicrobiana do O OE de M. palustris para todas as cepas Gram positivas testadas (B. subtilis, E. faecalis e S. aureus) com exceção de S. epidermidis. Em relação às Gram negativas apresentou atividade inibitória para S. flexneri. Foi demonstrada sua capacidade de sequestrar os radicais livres (DPPH) apresentando atividade antioxidante de até $82,81 \%$.

Esses resultados apontam um importante potencial para o uso desta planta como agente antimicrobiano e antioxidante, além de auxiliem para futuros estudos sobre atividades biológicas deste óleo essencial. Ressalta-se a importância de maiores estudos, principalmente referente a seus compostos, isolando-os e testando-os separadamente para avaliar outras possíveis aplicações uma futura utilização em diversos setores ambiental, agrícola e/ou industrial.

\section{Agradecimentos}

Agradecemos ao Conselho Nacional de Desenvolvimento Cientítico e Tecnológico (CNPq) e Fundação Araucária pelo financiamento da pesquisa, a Coordenação de Aperfeiçoamento de Pessoal de Nível Superior (CAPES) pela concessão da bolsa de mestrado e ao Programa de Pós-graduação em Manejo e Conservação de Recursos Naturais (PPRN) e ao Herbário UNOP pela identificação do exemplar voucher.

\section{Referências}

Adams, R. P. (2007). Identification of essential oil componentes by gas chromatography/Mass spectroscopy. $\left(4^{\circ}\right)$. Allured publishing corporation, USA.

Almeida, M. P., Romero, R.B., Romero, A. L. \& Crespan, E. R. (2015). Explorando a química e a atividade antifúngica de óleos essenciais: Uma proposta de projeto para a Educação Básica. Latin American Journal of Science Education, 1, 1-14.

Amorati, R., Foti, M. C. \& Valgimigli, L. (2013). Antioxidant Activity of Essential Oils. Journal of Agricultural and Food Chemistry, 61(46), 10835-10847. https://doi.org/10.1021/jf403496k

Amorim, B. S., Vasconcelos, T. N. C., Souza, G., Alves, M., Antonelli, A. \& Lucas, E. (2019). Advanced understanding of phylogenetic relationships, morphological evolution and biogeographic history of the mega-diverse plant genus Myrcia and its relatives (Myrtaceae: Myrteae). Molecular Phylogenetics and Evolution, 138, 65-88. https://doi.org/10.1016/j.ympev.2019.05.014

Babushok, V. I., Linstrom, P. J. \& Zenkevich, I. G. (2011). Retention Indices for Frequently Reported Compounds of Plant Essential Oils. Journal of Physical and Chemical Reference Data, 40(4), 043101. https://doi.org/10.1063/1.3653552

Becker, N. A., Volcão, L. M., Camargo, T. M., Freitag, R. A. \& Ribeiro, G. A. (2017). Biological properties of Eugenia uniflora L. essential oil: Chemical composition and antimicrobial activity. VITTALLE - Revista de Ciências Da Saúde, 29(1), 22-30. https://doi.org/10.14295/vittalle.v29i1.6267

Bozin, B., Mimica-Dukic, N., Samojlik, I. \& Jovin, E. (2007). Antimicrobial and Antioxidant Properties of Rosemary and Sage ( Rosmarinus officinalis L . and Salvia officinalis L ., Lamiaceae ) Essential Oils. Journal of Agricultural and Food Chemistry, 55, 7879-7885.

BRASIL. (2019). Farmacopéia Brasileira. RDC no 298 de 12 de agosto de 2019.

Castro, R. D. \& Lima, E. O. (2010). Atividade antifúngica in vitro do óleo essencial de Eucalyptus globulus L. sobre Candida spp. Rev. Odontol. UNESP (Online), 39(3), 179-184. https://revodontolunesp.com.br/article/588018b07f8c9d0a098b4d7f/pdf/rou-39-3-179.pdf

Cerqueira, M. D., Marques, E. J., Martins, D., Roque, N. F. \& Cruz, F. G. (2009). Variação sazonal da composição do oléo essencial de Myrcia salzmannii Berg. (Myrtaceae). Quimica Nova, 32(6), 1544-1548.

Cerqueira, M. D., Souza-Neta, L. C., Passos, M. G. V. M., Lima, E. de O., Roque, N. F., Martins, D., Guedes, M. L. S. \& Cruz, F. G. (2007). Seasonal variation and antimicrobial activity of Myrcia myrtifolia essential oils. Journal of the Brazilian Chemical Society, 18(5), 998-1003. https://doi.org/10.1590/S0103-50532007000500018

Claeson, P., Rådström, P., Sköld, O., Nilsson, Å. \& Höglund, S. (1992). Bactericidal effect of the sesquiterpene T-cadinol on Staphylococcus aureus. Phytotherapy Research, 6(2), 94-98. https://doi.org/10.1002/ptr.2650060209

CLSI. (2015). M07-10: Methods for diluition antimicrobial susceptibility tests for bacteria that grow aerobically; Approved Standard - Tenth Edition. Clinical and Laboratory Standards Institute, 35(2). https://clsi.org/media/1632/m07a10_sample.pdf 
Degáspari, C. H. \& Waszczynskyj, N. (2004). Propriedades antioxidantes de compostos fenólicos. Visão Acadêmica, 5(1). https://doi.org/10.5380/acd.v5i1.540

Gobbo-Neto, L. \& Lopes, N. P. (2007). Plantas medicinais: fatores de influência no conteúdo de metabólitos secundários. Química Nova, 30(2), 374-381. https://doi.org/10.1590/S0100-40422007000200026

Gonçalves, J. H. T., Santos, A. S. \& Morais, H. A. (2015). Atividade antioxidante, compostos fenólicos totais e triagem fitoquímica de ervas condimentares desidratadas. Revista Da Universidade Vale Do Rio Verde, 13(1), 486-497. https://doi.org/10.5892/ruvrd.v13i1.2003

Henriques, A. T., Sobral, M., Bridi, R., Vérin; P., Menut, C., Lamaty, G. \& Bessière, J. M. (1997). Essential Oils from Five Southern Brazilian Species of Myrcia (Myrtaceae). Journal of Essential Oil Research, 9(1), 13-18. https://doi.org/10.1080/10412905.1997.9700707

Jiménez, D., Araque, M., Rojas, L., Cordero, A. \& Briceño, B. (2012). Volatile components and antibacterial activity from Myrcia splendens (Sw.) DC. Rev. Fac. Farm., 54, 7-11.

Lang, G. \& Buchbauer, G. (2012). A review on recent research results (2008-2010) on essential oils as antimicrobials and antifungals. A review. Flavour and Fragrance Journal, 27(1), 13-39. https://doi.org/10.1002/ffj.2082

Limberger, R. P., Sobral, M., Henriques, A. T., Menut, C. \& Bessière, J.M. (2004). Óleos voláteis de espécies de Myrcia nativas do Rio Grande do Sul. Química Nova, 27(6), 916-919. https://doi.org/10.1590/S0100-40422004000600015

Lin, J., Dou, J., Xu, J. \& Aisa, H. A. (2012). Chemical Composition, Antimicrobial and Antitumor Activities of the Essential Oils and Crude Extracts of Euphorbia macrorrhiza. Molecules, 17(5), 5030-5039. https://doi.org/10.3390/molecules17055030

Machado, B. F. M. T., \& Junior, M. A. F. (2011). Óleos essenciais: aspectos gerais e usos em terapias naturais. Cadernos Acadêmicos, 3(2), 105-127. http://www.portaldeperiodicos.unisul.br/index.php/Cadernos_Academicos/article/view/718/671

Magina, M. A., Gilioli, A., Moresco, H. H., Colla, G., PizzolattiI, M. G. \& Brighente, I. M. C. (2010). Atividade antioxidante de três espécies de Eugenia (Myrtaceae). Latin American Journal of Pharmacy, 29(2), 376-382.

Melo, P. S., Bergamaschi, K. B., Tiveron, A. P., Massarioli, A. P., Oldoni, T. L. C.; Zanus, M. C., Pereira, G. E. \& Alencar, S. M. de. (2011). Composição fenólica e atividade antioxidante de resíduos agroindustriais. Ciência Rural, 4l(6), 1088-1093. https://doi.org/10.1590/S0103-84782011000600027

Mimica-Dukic, N.; Bozin, B.; Sokovic, M. \& Simin, N. (2004). Antimicrobial and Antioxidant Activities of Melissa officinalis L. (Lamiaceae) Essential Oil. Journal of Agricultural and Food Chemistry, 52(9), 2485-2489. https://doi.org/10.1021/jf030698a

Morais, S. M., Cavalcanti, E. S. B., Costa, S. M. O. \& Aguiar, L. A. (2009). Ação antioxidante de chás e condimentos de grande consumo no Brasil. Revista Brasileira de Farmacognosia, 19(1b), 315-320. https://doi.org/10.1590/S0102-695X2009000200023

Nascimento, P. F. C., Nascimento, A. C., Rodrigues, C. S., Antoniolli, Â. R., Santos, P. O., Barbosa Júnior, A. M. \& Trindade, R. C. (2007). Atividade antimicrobiana dos óleos essenciais: uma abordagem multifatorial dos métodos. Revista Brasileira de Farmacognosia, 17(1), 108-113. https://doi.org/10.1590/S0102-695X2007000100020

Ostrosky, E. A., Mizumoto, M. K., Lima, M. E. L., Kaneko, T. M., Nishikawa, S. O. \& Freitas, B. R. (2008). Métodos para avaliação da atividade antimicrobiana e determinação da Concentração Mínima Inibitória (CMI) de plantas medicinais. Revista Brasileira de Farmacognosia, 18(2), 301-307. https://doi.org/10.1590/S0102-695X2008000200026

Packer, J. F. \& Luz, M. M. S. (2007). Método para avaliação e pesquisa da atividade antimicrobiana de produtos de origem natural. Revista Brasileira de Farmacognosia, 17(1), 102-107. https://doi.org/10.1590/S0102-695X2007000100019

Pereira, A. S., Shitsuka, D. M., Parreira, F. J., \& Shitsuka, R. (2018). Metodologia da Pesquisa Científica. [e-book]. (1ª ed). Santa Maria: UAB/NTE/UFSM. Disponível em: https://repositorio.ufsm.br/bitstream/handle/1/15824/Lic_Computacao_Metodologia-Pesquisa -Cientifica.pdf.sequence=1. Acesso em: $28 \mathrm{fev}$. 2021

Pandini, J. A., Gisele, F. G. S., Scur, M. C., Francisco, L., Alves, A. \& Martins, C. C. (2015). Antimicrobial , insecticidal , and antioxidant activity of essential oil and extracts of Guarea kunthiana A . Juss. Journal of Medicinal Planta Research, 9(3), 48-55. https://doi.org/10.5897/JMPR2014.5551

Rice-Evans, C. A., Miller, N. J. \& Paganga, G. (1996). Structure-antioxidant activity relationships of flavonoids and phenolic acids. Free Radical Biology and Medicine, 20(7), 933-956. https://doi.org/10.1016/0891-5849(95)02227-9

Rosa, C. S., Veras, K. S., Silva, P. R., Lopes Neto, J. J.; Cardoso, H. L. M.; Alves, L. P. L.; Brito, M. C. A.; Amaral, F. M. M.; Maia, J. G. S.; Monteiro, O. S. \& Moraes, D. F. C. (2016). Composição química e toxicidade frente Aedes aegypti L. e Artemia salina Leach do óleo essencial das folhas de Myrcia sylvatica (G. Mey.) DC. Revista Brasileira de Plantas Medicinais, 18(1), 19-26. https://doi.org/10.1590/1983-084X/15_006

Rufino, M. do S. M., Alves, R. E., Brito, E. S., Morais, S. M., Sampaio, C. de G., Pérez-Jiménez, J. \& Fulgencio Diego Saura-Calixto. (2007). Metodologia Científica: Determinação da Atividade Antioxidante Total em Frutas pela Captura do Radical Livre DPPH. Comunicado Técnico Embrapa, 1-4.

Russo, E. M. K., Reichelt, A. A. J., Sá, J. R., Furlanetto, R. P., Moises, R. C. S., Kasamatsu, T. S. \& Chacra, A. R. (1990). Clinical trial of Myrcia uniflora and Bauhinia forficata leaf extracts in normal and diabetic patients. Brazilian Journal of Medical and Biological Research, 23(1), 11-20.

Sá, F. A. S., Borges, L. L., Paula, J. A. M., Sampaio, B. L., Ferri, P. H. \& Paula, J. R. (2012). Essential oils in aerial parts of Myrcia tomentosa: composition and variability. Revista Brasileira de Farmacognosia, 22(6), 1233-1240. https://doi.org/10.1590/S0102-695X2012005000120

Santana, C. B., Souza, J. G. de L., Coracini, M. D. A., Walerius, A. H., Soares, V. D., Costa, W. F. da \& Pinto, F. G. da S. (2018). Chemical composition of essential oil from Myrcia oblongata DC and potencial antimicrobial, antioxidant and acaricidal activity against Dermanyssus gallinae (Degeer, 1778). Bioscience Journal, 34(4), 996-1009. https://doi.org/10.14393/BJ-v34n1a2018-39599 
Santos, J. R. N., Teles, A. M., Ferreira, C. G. \& Mouchrek, A. N. (2020). Avaliação da atividade bactericida e antioxidante do óleo essencial e do extrato hidroalcoólico de orégano (Origanum vulgare). Research, Society and Development, 9(10), e7829108410. https://doi.org/10.33448/rsd-v9i10.8410

Sartoratto, A., Machado, A. L. M., Delarmelina, C., Figueira, G. M., Duarte, M. C. T. \& Rehder, V. L. G. (2004). Composition and antimicrobial activity of essential oils from aromatic plants used in Brazil. Brazilian Journal of Microbiology, 35(4), 275-280. https://doi.org/10.1590/S1517-83822004000300001

Scherer, R., Wagner, R., Duarte, M. C. T. \& Godoy, H. T. (2009). Composição e atividades antioxidante e antimicrobiana dos óleos essenciais de cravo-daíndia, citronela e palmarosa. Revista Brasileira de Plantas Medicinais, 11(4), 442-449. https://doi.org/10.1590/S1516-05722009000400013

Scur, M. C., Pinto, F. G. da S., Bona, E. A. M., Weber, L. D., Alves, L. F. A. \& Moura, A. C. de. (2014). Occurrence and antimicrobial resistance of Salmonella serotypes isolates recovered from poultry of Western Paran, Brazil. African Journal of Agricultural Research, 9(9), 823-830. https://doi.org/10.5897/AJAR2013.8202

Silva, A. C., Iacuzio, R., Da Silva Cândido, T. J., Xavier Rodrigues, M. \& Cirone Silva, N. C. (2018). Resistência antimicrobiana de Salmonella spp., Staphylococcus aureus E Escherichia coli isolados de carcaças de frangos: Resistência a antibióticos e óleos essenciais. Revista Brasileira de Agropecuária Sustentável, 8(1), 95-103. https://doi.org/10.21206/rbas.v8i1.474

Silva, J. G., Souza, I. A., Higino, J. S., Siqueira-Junior, J. P., Pereira, J. V. \& Pereira, M. do S. V. (2007). Atividade antimicrobiana do extrato de Anacardium occidentale Linn. em amostras multiresistentes de Staphylococcus aureus. Revista Brasileira de Farmacognosia, $17(4), 572-577$. https://doi.org/10.1590/S0102-695X2007000400016

Solórzano-Santos, F. \& Miranda-Novales, M. G. (2012). Essential oils from aromatic herbs as antimicrobial agents. Current Opinion in Biotechnology, 23(2), 136-141. https://doi.org/10.1016/j.copbio.2011.08.005

Souza, T. J. T., Apel, M. A., Bordignon, S., Matzenbacher, N. I., Zuanazzi, J. Â. S. \& Henriques, A. T. (2007). Composição química e atividade antioxidante do óleo volátil de Eupatorium polystachyum DC. Revista Brasileira de Farmacognosia, 17(3), 368-372. https://doi.org/10.1590/S0102-695X2007000300011

Stefanello, M. É. A., Pascoal, A. C. R. F. \& Salvador, M. J. (2011). Essential Oils from Neotropical Myrtaceae: Chemical Diversity and Biological Properties. Chemistry \& Biodiversity, 8(1), 73-94. https://doi.org/10.1002/cbdv.201000098

Takao, L. K., Imatomi, M. \& Gualtieri, S. C. J. (2015). Antioxidant activity and phenolic content of leaf infusions of Myrtaceae species from Cerrado (Brazilian Savanna). Brazilian Journal of Biology, 75(4), 948-952.

Weber, L. D., Pinto, F. G. da S., Scur, M. C., Souza, J. G. L., Costa, W. F. \& Leite, C. W. (2014). Chemical composition and antimicrobial and antioxidant activity of essential oil and various plant extracts from Prunus myrtifolia (L.) Urb. African Journal of Agricultural Research, 9(9), 846-853. https://doi.org/10.5897/AJAR2013.8260

Xavier, M., Alves, J., Carneiro, N., Souchie, E., Silva, E., Martins, C., Ambrósio, M., Egea, M., Alves, C. \& Miranda, M. (2016). Composição química do óleo essencial de Cardiopetalum calophyllum Schltdl. (Annonaceae) e suas atividades antioxidante, antibacteriana e antifúngica. Revista Virtual de Química, 8(5).

Yu, J. Q., Liao, Z. X., Cai, X. Q., Lei, J. C. \& Zou, G. L. (2007). Composition, antimicrobial activity and cytotoxicity of essential oils from Aristolochia mollissima. Environmental Toxicology and Pharmacology, 23(2), 162-167. https://doi.org/10.1016/j.etap.2006.08.004 